\title{
Predicting functional outcome in acute stroke: comparison of a simple six variable model with other predictive systems and informal clinical prediction
}

\author{
C Counsell, M Dennis, M McDowall
}

See Editorial Commentary, p 351

J Neurol Neurosurg Psychiatry 2004;75:401-405. doi: 10.1136/jnnp.2003.018085

Background: Statistical models that predict functional outcome after stroke using six simple variables (SSV) have recently been developed and validated.

See end of article for authors' affiliations

.............

Correspondence to: Dr Carl Counsell, Department of Medicine and Therapeutics, University of Aberdeen, Polwarth Building, Foresterhill, Aberdeen AB25 2ZD, UK : cec@iahs.abdn.ac.uk

Received 22 May 2003 In revised form 7 August 2003

Accepted 8 August 2003
Objective: To compare the accuracy of these models with other simple ways of predicting outcome soon after stroke.

Methods: The SSV model for being alive and independent (modified Rankin score $\leqslant 2$ ) six months or one year after stroke was compared with predictions based on a model that included only age and Oxford community stroke project classification, with predictions based on conscious level and urinary continence, and with informal clinical predictions made by clinicians interested in stroke. Predictions were compared in an independent hospital based cohort of stroke patients using receiver operator characteristic (ROC) curves.

Results: The SSV model at six months had a significantly greater area under the curve (0.84) than the model with only age and stroke classification (0.75). Predictions based on conscious level and urinary continence were no better than those of the SSV model and were unable to predict subjects with a high probability of good outcome. The sensitivity and specificity for informal clinical predictions at one year lay on or below the SSV model curve, implying that the SSV model was at least as good as clinical predictions. Conclusions: The SSV models performed as well as or better than other simple predictive systems. These models will be useful in epidemiological studies but should not be used to guide clinical management until their impact on patient care and outcome has been evaluated.
A ccurate prediction of outcome in the acute and subacute phase of stroke would have several uses in both epidemiological research and clinical practice. ${ }^{1}$ We have recently described the development and validation of logistic regression models that use six simple variables (table 1), collected shortly after the onset of ischaemic or haemorrhagic stroke, to predict the probability of a patient being alive and independent at six or 12 months. ${ }^{1-3}$ We wished to test whether these new models gave more accurate predictions than other predictive systems that have been developed, and whether they were as accurate as informal predictions of outcome made by clinicians looking after stroke patients. The latter may be better than any statistical model because clinicians can take the whole clinical picture into account as well their previous experience and intuition.

\section{METHODS}

Initially, we hoped to compare the six simple variables (SSV) models with other models from a recent systematic review ${ }^{4}$ that predicted functional outcome at six to 12 months. However, this was not possible because all except one of the previous models included some variables that were not collected in the cohort that we used to compare the models. The remaining model was described in insufficient detail for it to be used. We therefore compared the SSV models with two other simple predictive systems: first, a model that included only two variables, age and the Oxfordshire community stroke project (OCSP) classification of stroke syndrome (which has been used previously to provide a simple assessment of prognosis $\left.{ }^{5-7}\right)$; and second, predictions based on two variables (conscious level and urinary continence) which others have suggested give as good a prediction as those based on more complex predictive models. ${ }^{89}$ We also tested how the SSV models compared with early informal clinical predictions of outcome made by physicians of varying stroke experience.

The various prediction methods were compared in the first 1330 patients from the Lothian stroke register (LSR), an independent hospital based cohort of patients (that is, it was not used to generate any of the models), in which the median time from stroke onset to patient assessment was 2.8 days (interquartile range 1 to 7 days). " "Alive and independent" was defined as a modified Rankin score of 2 or less.

\section{Prediction of outcome using age and OCSP classification}

A logistic regression model for being alive and independent at six months using only these two variables was produced on the same 530 patients in the OCSP cohort who were used to generate the original SSV models. This new model was then validated on 1200 patients in the LSR cohort ( 130 had missing outcome data) and its area under the receiver operator characteristic (ROC) curve was compared with that of the SSV model as previously described. ${ }^{1}$

\section{Prediction of outcome using conscious level and urinary continence}

Data on conscious level, urinary continence, and six months outcome were available for 1187 of the 1330 patients in the LSR. Previous investigators ${ }^{89}$ have suggested that a simple

Abbreviations: GCS, Glasgow coma scale; LSR, Lothian stroke register; OCSP, Oxfordshire community stroke project; ROC, receiver operator characteristic; SSV, six simple variables model 
Table 1 Six simple variables for predicting outcome after stroke

\begin{tabular}{|c|c|c|c|}
\hline \multirow[b]{2}{*}{ Variable* } & \multirow[b]{2}{*}{ Definition of variable } & \multicolumn{2}{|c|}{ OR for being alive and independent $(95 \% \mathrm{CI})$} \\
\hline & & At six months & At one year \\
\hline $\begin{array}{l}\text { Age } \\
\text { Living alone } \\
\text { Independent pre-stroke } \\
\text { Normal GCS verbal } \\
\text { Able to lift arms } \\
\text { Able to walk }\end{array}$ & $\begin{array}{l}\text { Age at onset (years) } \\
\text { Nobody permanently living with } \\
\text { patient before stroke } \\
\text { Modified Rankin score } \leqslant 2 \\
\text { before stroke } \\
\text { GCS verbal score }=5 \text { (that is, } \\
\text { oriented) } \\
\text { Able to lift both arms to horizontal } \\
\text { (MRC score } \geqslant 3 \text { in both arms) } \\
\text { Walks without the help of another } \\
\text { person (can use stick/frame) }\end{array}$ & $\begin{array}{l}0.95+(0.93 \text { to } 0.97) \\
0.52(0.31 \text { to } 0.86) \\
15.55(5.68 \text { to } 42.58) \\
8.67(3.43 \text { to } 21.91) \\
8.22(3.25 \text { to } 20.76) \\
3.71(1.77 \text { to } 7.77)\end{array}$ & $\begin{array}{l}0.92 \dagger(0.89 \text { to } 0.94) \\
0.68(0.41 \text { to } 1.13) \\
23.90(6.83 \text { to } 83.63) \\
8.82(3.28 \text { to } 23.70) \\
10.16(3.72 \text { to } 27.78) \\
3.17(1.44 \text { to } 6.97)\end{array}$ \\
\hline
\end{tabular}

classification of alert versus drowsy/comatose provided good predictions of short term outcome. Unfortunately, conscious level in patients in the LSR was only defined in terms of the Glasgow coma scale (GCS) rather than as being alert or not. We therefore reclassified patients in the LSR as "alert" if their initial GCS was $15 / 15$ in those without dysphasia or a combined motor and eye component of 10/10 in those with dysphasia. The remaining patients were classified as drowsy or comatose. Urinary incontinence was defined as any episode of incontinence-patients with a catheter or penile sheath were classified as incontinent. The sensitivity and specificity for the four possible categories of conscious level and continence (alert versus not, continent versus not, alert and continent versus not, alert or continent versus neither) were used to plot an ROC curve for alive and independent at six months. These curves were then compared qualitatively to the ROC curves of the SSV model. Formal statistical testing of the difference in areas under the ROC curves was not undertaken because there were so few points for conscious level and continence.

\section{Informal clinical prediction of outcome}

All patients in the LSR were seen by one of six neurology trainees or three consultants, who all had a special interest in stroke. After they had seen the patient, each clinician used their clinical judgement to predict the patient's modified Rankin score at one year. The clinical forecasts were made after the same assessment of the patient that was used to collect the baseline data for the SSV model; they were thus directly comparable with the model's predictions. However, none of the forecasts was made using the SSV models and most ( 1071 of $1330,80 \%$ ) were made before the model was developed.

\section{Analyses}

The patient's actual status was established by telephone follow up by staff blind to the baseline clinical and model prediction. The level of agreement over that expected by chance between the informal clinical prediction and the actual outcome was assessed by calculating a weighted $\kappa$ value. ${ }^{10}$ The sensitivity, specificity, and positive and negative predictive values of the clinical predictions were calculated (plus 95\% confidence intervals) and plotted on the ROC curve for the validated SSV model for alive and independent at one year. As we had previously shown that the SSV model had much poorer discrimination in outpatients than in inpatients, performance of the informal clinical predictions was analysed in outpatients and inpatients separately. We were unable to assess the relative performance of predictions made by consultants versus trainees, because the person who made the prediction was not entered into the database for most patients. However, most predictions for inpatients were made by trainees while most outpatient predictions were made by consultants.

\section{RESULTS \\ Prediction of outcome using age and OCSP classification}

The validation showed that the model including only age and stroke syndrome had a significantly smaller $(2 p<0.0001)$ area under the ROC curve than the corresponding SSV model, implying worse discrimination (fig l).

\section{Prediction of outcome using conscious level and urinary continence}

The sensitivities for predicting a good outcome were high for all categories $(>0.90)$ (that is, very few patients who had a good outcome were initially not alert or continent), but the specificities were low $(<0.5)$ (table 2 ). Urinary continence alone had a significantly greater specificity than conscious level alone. Figure 2 shows that the points for conscious level and continence all lie below the ROC curve for the SSV

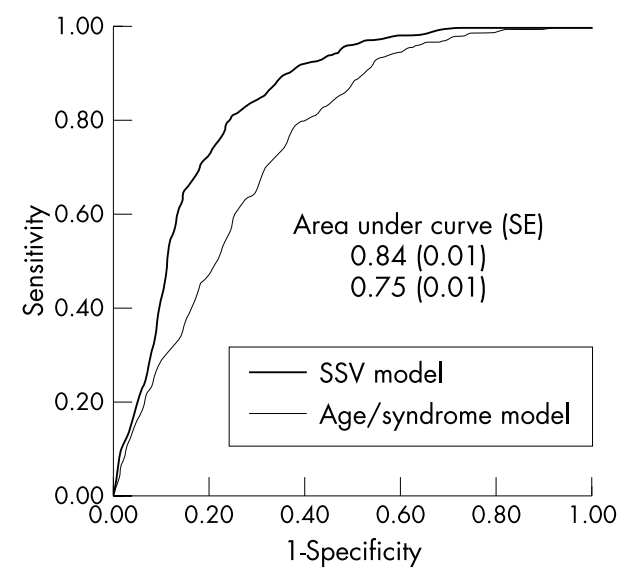

Figure 1 Receiver operator characteristic curves for prediction of being alive and independent at six months. Simple six variable (SSV) model $v$ model including only age and stroke syndrome: log lodds of independent survival $)=-2.61-[0.07 \times$ age in years $]+[3.44 \times \mathrm{TACS}]+[0.77 \times \mathrm{PACS}]+[0.11 \times \mathrm{POCS}]$, where TACS, PACS, and POCS scored 1 if present and 2 if absent. PACS, partial anterior circulatory stroke syndrome; POCS, posterior circulatory stroke syndrome; TACS, total anterior circulatory stroke syndrome. 
Table 2 Sensitivity and specificity of conscious level and urinary continence in predicting good functional outcome at six months in the Lothian stroke register

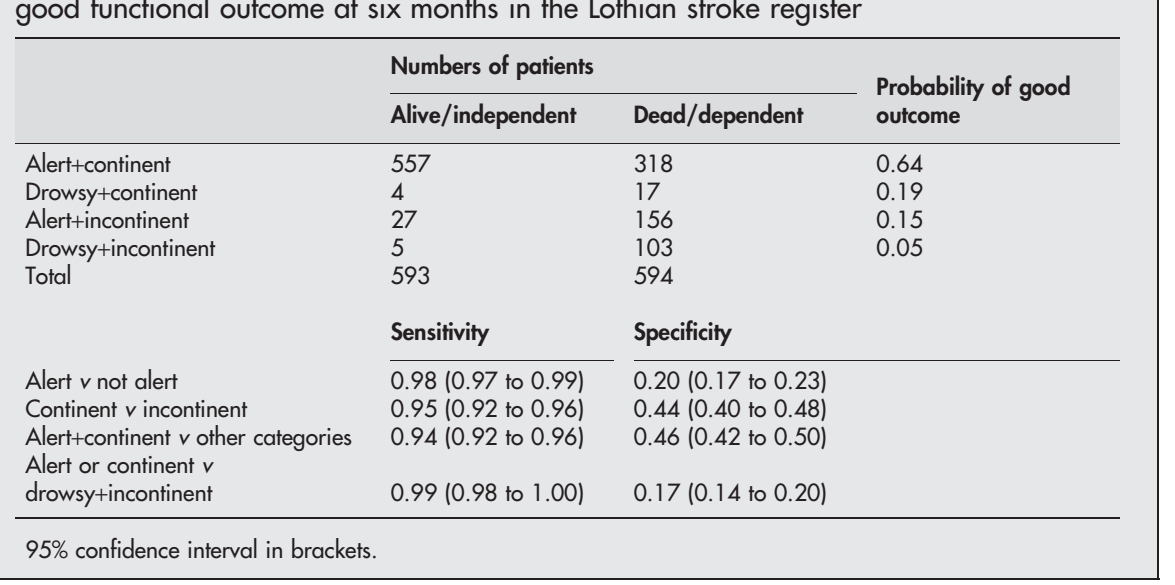

model, implying worse prediction, although the differences were small. However, the combination of conscious level and continence gave very limited information compared with SSV model because it only provided four categories which clustered close together on the ROC curve. This meant that it was not possible to identify patients with a high probability of a good outcome using just conscious level and continence: patients who were both alert and continent still had a probability of a good outcome of only 0.64 (table 2 ).

\section{Informal clinical prediction of outcome}

Clinical predictions and one year outcome data were available for 1287 patients in LSR (952 inpatients, 335 outpatients). The agreement between the clinical predictions and actual outcomes is shown in table 3, and data on the predictive accuracy of clinical predictions are given in table 4 . The level of agreement for clinical predictions was moderate for inpatients (weighted $\kappa$ 0.44) and fair for outpatients (weighted $\kappa 0.25$ ). Clinicians tended to be overoptimistic in their prediction of good outcome for inpatients: only $65 \%$ of those predicted to be alive and independent at one year actually were independent, whereas $90 \%$ of those predicted to do badly were dead or dependent at one year. For both inpatients and outpatients, the sensitivity and specificity of the informal clinical predictions lay on or below the ROC curve for the SSV model, implying that the model performed at least as well as the clinical predictions (fig 3).

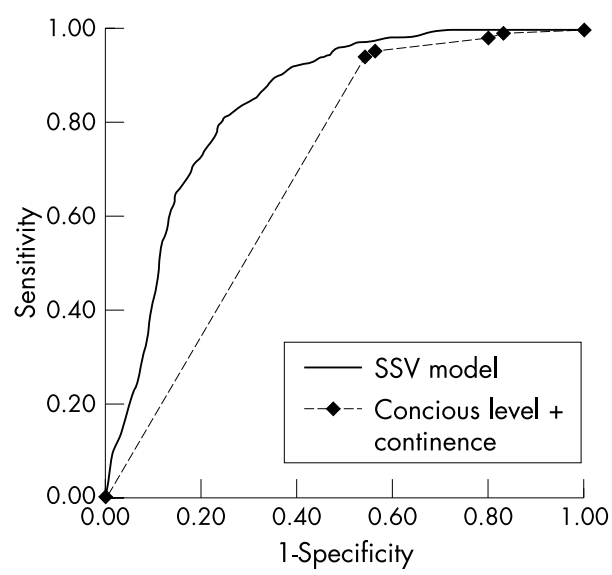

Figure 2 Receiver operator characteristic curves for prediction of being alive and independent at six months: simple six variable model (SSV) $v$ conscious level and urinary continence.

\section{DISCUSSION}

We have compared our simple model with other simple predictive systems but unfortunately were unable to compare the new SSV model with more complex models because many of the variables from other models were not collected in the LSR. Such comparisons will require specific prospective studies. It may be argued that to obtain accurate predictions of outcome-particularly for use in clinical practice-more complex models with detailed clinical and laboratory data are required. However, we have previously shown that more detailed models do not necessarily provide better predictions for a variety of reasons. ${ }^{1}$ Moreover, complex models will not be feasible for epidemiological research such as comparison of outcomes in different cohorts or stratification in clinical trials.

The SSV model had better discrimination (greater area under the ROC curve) than the model that included age and OCSP stroke syndrome. This is not surprising as the OCSP system was primarily designed to classify different types of ischaemic stroke based on the pattern of neurological deficit rather than to assess prognosis, although it has been used to do the latter. ${ }^{6}{ }^{7}$ The syndrome model did not include variables related to the premorbid condition of the patient which can influence prognosis, and takes no account of the severity of a neurological deficit-for example, the lacunar syndrome does not differentiate between a patient with a mild hemiparesis or a complete hemiplegia. Finally, the OCSP classification is less reliable than the variables included in the SSV model. It has been shown to have a $\kappa$ value of about 0.5 compared with values of 0.7 or more for the six variables in the SSV model. ${ }^{11}{ }^{12}$ Superficially, a model including only age and stroke syndrome may appear to be easier to use than one including six variables. However, in reality it would be more difficult to use. In order to classify the stroke syndrome, a detailed neurological examination is required. By comparison, the six simple variables can be collected by a short history and examination of arm and leg function only, which could probably be done by non-medically-trained observers.

Predictions based on conscious level and continence, although simple, were usually not as good as those of the SSV model. It was possible to identify those with a very low probability of being alive and independent (patients who were incontinent and drowsy) using this system, but not those with a high probability of a good outcome. The latter can be achieved with the SSV model. Other problems with this predictive system include a lack of clinical credibility ${ }^{13}$ (for example, it does not seem sensible to predict a person's disability status after a stroke without taking their pre-stroke disability into account) and the fact that the assessment of 
Table 3 Numbers of patients subdivided by predicted (informally by clinicians) and actual modified Rankin score at one year

\begin{tabular}{|c|c|c|c|c|c|c|c|c|c|}
\hline & & \multicolumn{8}{|c|}{ Actual modified Rankin score at one year } \\
\hline & & 0 & 1 & 2 & 3 & 4 & 5 & 6 & Total \\
\hline \multicolumn{10}{|l|}{ Inpatients } \\
\hline Predicted modified & 0 & 7 & 22 & 14 & 3 & 0 & 0 & 2 & 48 \\
\hline \multirow[t]{7}{*}{ Rankin score } & 1 & 24 & 37 & 73 & 29 & 7 & 3 & 10 & 183 \\
\hline & 2 & 21 & 27 & 70 & 54 & 10 & 6 & 34 & 222 \\
\hline & 3 & 3 & 12 & 27 & 57 & 35 & 22 & 54 & 210 \\
\hline & 4 & 0 & 2 & 5 & 21 & 16 & 19 & 51 & 114 \\
\hline & 5 & 0 & $\overline{0}$ & 1 & 1 & 4 & 9 & 20 & 35 \\
\hline & 6 & 1 & 0 & 2 & 8 & 9 & 9 & 111 & 140 \\
\hline & Total & 56 & 100 & 192 & 173 & 81 & 68 & 282 & 952 \\
\hline \multicolumn{10}{|l|}{ Outpatients } \\
\hline Predicted modified & 0 & 24 & 20 & 10 & 1 & 1 & 0 & 1 & 57 \\
\hline \multirow[t]{7}{*}{ Rankin score } & 1 & 42 & 55 & 44 & 14 & 0 & 1 & 4 & 160 \\
\hline & 2 & 8 & 23 & 42 & 16 & 4 & 0 & 8 & 101 \\
\hline & 3 & 0 & 1 & 5 & 4 & 1 & 1 & 0 & 12 \\
\hline & 4 & 0 & 0 & 0 & 2 & 0 & 0 & 0 & 2 \\
\hline & 5 & 0 & 0 & 0 & 0 & 0 & 0 & 0 & 0 \\
\hline & 6 & 0 & 0 & 1 & 0 & 0 & 1 & 1 & 3 \\
\hline & Total & 74 & 99 & 102 & 37 & 6 & 3 & 14 & 335 \\
\hline
\end{tabular}

conscious level (alert $v$ drowsy) has poorer inter-rater reliability than the variables in the SSV model. ${ }^{12}$ Although the LSR did not contain data on conscious level as defined by previous investigators (alert $v$ not alert), we felt that our reclassification on the basis of the GCS was unlikely to have introduced significant bias.

We believe this is the first study that has compared a statistical prediction of outcome following stroke with clinicians' informal predictions based on clinical judgement. This is important because before statistical predictive models can be used in clinical practice they should be shown to be as good as or better than existing informal clinical predictions that clinicians use all the time to make management decisions. ${ }^{13}$ The informal clinical prediction, although flawed, appeared to be as good as prediction with the SSV model based on the ROC curves. This may imply that the SSV model would have only a small role to play in routine clinical practice. However, because the clinical predictions were dichotomised into those that were correct and those that were incorrect, we were only able to assess their accuracy at one point on the ROC curve. It is possible that clinical predictions may be better or worse than the model's predictions at other points on the curve. The only way to assess this would be to ask clinicians to provide a probability of a good outcome in each patient at baseline which would allow an ROC curve to be drawn and compared with the ROC curve of the model.

The informal predictions in this study were made by clinicians with specific training and interest in stroke, although their experience varied. In many respects, it is therefore encouraging that the SSV model performed as well as their predictions. It is possible that clinicians with less

Table 4 Accuracy of informal clinical predictions of good outcome at one year in Lothian stroke register

\begin{tabular}{lll}
\hline & Inpatients & Outpatients \\
\hline Sensitivity & $0.85(0.81$ to 0.88$)$ & $0.97(0.95$ to 0.99$)$ \\
Specificity & $0.74(0.70$ to 0.77$)$ & $0.17(0.08$ to 0.28$)$ \\
Positive predictive value & $0.65(0.61$ to 0.70$)$ & $0.84(0.80$ to 0.88$)$ \\
Negative predictive value $0.89(0.87$ to 0.92$)$ & $0.59(0.33$ to 0.82$)$ \\
\hline 95\% confidence interval in brackets. & \\
\hline
\end{tabular}

expertise in stroke would have performed less well, but we were unable to test this. However, one study in patients with head injury did show that predictions of outcome made by more junior members of staff were less accurate than those made by consultants and senior registrars. ${ }^{14}$ If this were also the case in stroke, then clinicians with less expertise may find the SSV model useful in clinical practice. The models are also explicit in defining which factors are important in predicting outcome, whereas much of the informal prediction by experts is implicit. It may therefore be easier for doctors to improve their predictions by using the models rather than by trying to learn the art of informal clinical prediction from an expert.

\section{Conclusions}

We have shown that other simple predictive systems have significant disadvantages compared with our new simple models, which also seem to perform as well as informal clinical predictions made by clinicians with an interest in stroke. Further prospective studies are required to compare the SSV models with other available statistical models. This study strengthens our belief that the SSV models will be useful tools in epidemiological studies but we would argue against their use to guide clinical practice until their clinical

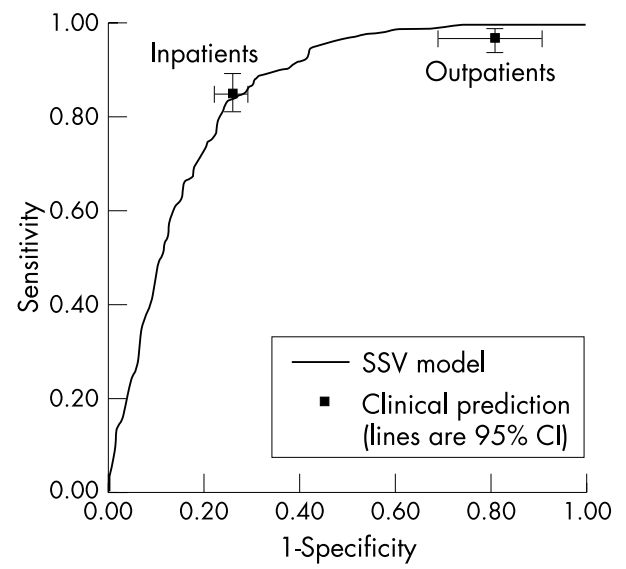

Figure 3 Comparison of informal clinical prediction with model prediction at one year. $\mathrm{Cl}$, confidence interval. 
utility has been evaluated, in particular to see if they improve patient care and outcomes. ${ }^{13}$

\section{Authors' affiliations}

C Counsell, Department of Medicine and Therapeutics, University of Aberdeen, Aberdeen, UK

M Dennis, M McDowall, Department of Clinical Neurosciences, Western General Hospital, Edinburgh, UK

Competing interests: none declared

\section{REFERENCES}

1 Counsell C, Dennis M, McDowall M, et al. Predicting outcome after acute and subacute stroke: development and validation of new prognostic models. Stroke 2002:33:1041-7.

2 The FOOD Trial Collaborators. Performance of a statistical model to predict stroke outcome in the context of a large, simple, randomized, controlled trial of feeding. Stroke 2003;34:127-33.

3 Warlow CP, Dennis MS, van Giin J, et al. Predicting outcome in individual patients. In: Stroke: a practical guide to management, 2 nd ed, section 10.2.7. Oxford: Blackwell Scientific, 2000:422-7.

4 Counsell C, Dennis M. A systematic review of prognostic models in acute stroke. Cerebrovascular Diseases 2001;12:159-70.
5 Bamford J, Sandercock P, Dennis M, et al. Classification and natural history of clinically identifiable subtypes of cerebral infarction. Lancet 1991;337:1521-6.

6 Dyker AG, Weir CJ, Lees KR. Influence of cholesterol on survival after stroke: retrospective study. BMJ 1997:314:1584-8.

7 Weir CJ, Murray GD, Dyker AG, et al. Is hyperglycaemia an independent predictor of poor outcome after acute stroke? Results of a long-term follow up study. BMJ 1997;314:1303-6.

8 Wade DT, Hewer RL. Outlook after an acute stroke: urinary incontinence and loss of consciousness compared in 532 patients. Q J Med 1985;56:601-8.

9 Gladman JR, Harwood DM, Barer DH. Predicting the outcome of acute stroke: prospective evaluation of five multivariate models and comparison with simple methods. J Neurol Neurosurg Psychiatry 1992;55:347-51.

10 Altman DG. Some common problems in medical research. In: Altman DG, ed. Practical statistics for medical research, 1 st ed. London: Chapman and Hall, 1993:396-439.

11 Lindley RI, Warlow CP, Wardlaw JM, et al. Interobserver reliability of a clinical classification of acute cerebral infarction. Stroke 1993;24:1801-4.

12 Weir N, Counsell C, McDowall M, et al. The reliability of the variables in a new set of models to predict outcome after stroke. I Neurol Neurosurgery Psychiatry 2003;74:447-51

13 Wyatt JC, Altman DG. Commentary: prognostic models: clinically useful or quickly forgotten? BMJ 1995;311:1539-41.

14 Barlow P, Teasdale G. Prediction of outcome and management of severe head injuries: the attitude of neurosurgeons. Neurosurgery 1986;19:989-91. 